\title{
Alone or With Others? Understanding Eating Episodes of College Students with Mobile Sensing
}

\author{
Lakmal Meegahapola \\ Idiap Research Institute and EPFL \\ Switzerland \\ lmeegahapola@idiap.ch
}

\author{
Salvador Ruiz-Correa \\ IPICYT \\ Mexico \\ salvador.ruiz@ipicyt.edu.mx
}

\author{
Daniel Gatica-Perez \\ Idiap Research Institute and EPFL \\ Switzerland \\ gatica@idiap.ch
}

\begin{abstract}
Understanding food consumption patterns and contexts using mobile sensing is fundamental to build mobile health applications that require minimal user interaction to generate mobile food diaries. Many available mobile food diaries, both commercial and in research, heavily rely on self-reports, and this dependency limits the long term adoption of these apps by people. The social context of eating (alone, with friends, with family, with a partner, etc.) is an important self-reported feature that influences aspects such as food type, psychological state while eating, and the amount of food, according to prior research in nutrition and behavioral sciences. In this work, we use two datasets regarding the everyday eating behavior of college students in two countries, namely Switzerland $\left(\mathrm{N}_{c h}=122\right)$ and Mexico $\left(\mathrm{N}_{m x}=84\right)$, to examine the relation between the social context of eating and passive sensing data from wearables and smartphones. Moreover, we design a classification task, namely inferring eating-alone vs. eating-with-others episodes using passive sensing data and time of eating, obtaining accuracies between $77 \%$ and $81 \%$. We believe that this is a first step towards understanding more complex social contexts related to food consumption using mobile sensing.
\end{abstract}

\section{CCS CONCEPTS}

- Human-centered computing $\rightarrow$ Mobile computing; Smartphones; Mobile phones; - Applied computing $\rightarrow$ Consumer health; Health informatics.

\section{KEYWORDS}

wearable sensing, mobile sensing, smartphone sensing, health, wellbeing, eating behavior, food diaries, social context, passive sensing

\section{ACM Reference Format:}

Lakmal Meegahapola, Salvador Ruiz-Correa, and Daniel Gatica-Perez. 2020. Alone or With Others? Understanding Eating Episodes of College Students with Mobile Sensing. In 19th International Conference on Mobile and Ubiquitous Multimedia (MUM 2020), November 22-25, 2020, Essen, Germany. ACM, New York, NY, USA, 5 pages. https://doi.org/10.1145/3428361.3428463

Permission to make digital or hard copies of all or part of this work for personal or classroom use is granted without fee provided that copies are not made or distributed for profit or commercial advantage and that copies bear this notice and the full citation on the first page. Copyrights for components of this work owned by others than the author(s) must be honored. Abstracting with credit is permitted. To copy otherwise, or republish, to post on servers or to redistribute to lists, requires prior specific permission and/or a fee. Request permissions from permissions@acm.org.

MUM 2020, November 22-25, 2020, Essen, Germany

(c) 2020 Copyright held by the owner/author(s). Publication rights licensed to ACM. ACM ISBN 978-1-4503-8870-2/20/11 .\$ \$15.00

https://doi.org/10.1145/3428361.3428463

\section{INTRODUCTION}

Food and Nutrition has risen as the second most common category of apps used by mHealth app users according to recent reports [27]. Most of these apps have already incorporated mobile food diaries/journals to provide basic temporal insights to users. Even though many food diary-based studies have been carried out in the past with encouraging results for food consumption related interventions [26, 42], passive smartphone sensing has just begun to be widely used, comparatively speaking, in conjunction with food diaries, to understand contextual aspects that affect food consumption [5, 33].

Mobile food diaries use two main techniques to capture data $[5,32,33]$. They are: (a) Passive Sensing - using embedded sensors in smartphones and wearables (accelerometer, gyroscope, location, etc.) and events generated from the phone (app usage, screen-on time, and battery charging events), models can unobtrusively generate behavioral and contextual insights; and (b) Self-Reports - capture details regarding daily behavior and context related to eating. According to prior literature in mobile sensing $[5,33]$ where eating is considered as a holistic event with interconnected dimensions $[6,37]$, the social context of eating is an important variable that is self-reported, as it is a factor that relates to many aspects regarding food consumption episodes such as location, time, psychological state while eating, physical conditions, and food amount.

Studies have found that eating in highly social contexts (partying, celebrations, gatherings, etc.) can influence the amount of food consumed, which might lead to overeating in the short term [7, $8,29]$ and to eating disorders in the long term [10, 35]. Further, concepts such as social facilitation and impression management emphasize how the presence of one or more people when eating can lead to overeating and undereating, respectively [17-19]. Moreover, studies have examined the effects of eating-alone and eating-withothers as fundamental aspects regarding eating behavior [19, 40]. Hence, understanding the social context of eating has been outlined as an important component of food consumption research $[9,11,12$, $14,16,37]$. Furthermore, automatically inferring attributes related to social context would enable mobile food diaries to send contextaware notifications [21] and to support interventions [30], and also to help users adhere to healthy eating practices $[14,20]$. In this study, similar to prior mHealth sensing studies with food diaries $[5,25,33]$, we consider eating to be a holistic event, and use a binary categorization for the social context of eating - eating-alone vs. eating-with-others as a construct to understand food consumption behavior of college students in two countries. Hence, this paper has two contributions:

Contribution 1: We conducted a data analysis of wearable and mobile sensing datasets collected from 206 college students of two 
countries (Switzerland and Mexico). We highlight behavioral differences in the two countries, and show that factors such as time, physical activity levels, and location around eating episodes, all of which are passively sensed via wearables or smartphones, are features that help discriminate the social context of eating.

Contribution 2: We define and evaluate a classification task related to inferring the social context of eating (eating-alone vs. eatingwith-others) using time of eating and passive sensing (wearable and smartphone) data related to physical activity levels and eating contexts, with accuracies in the range $77 \%-81 \%$. These accuracies increase further when combined with other contextual features in both datasets. Hence, these initial results suggest that self-reported social context of eating can be inferred using sensing data with a above-baseline accuracy, showing the potential of using passive sensing together with mobile food diaries towards understanding more complex social contexts of eating.

\section{DATASETS AND PRE-PROCESSING}

The feature groups we used are temporal (T), contextual $(\mathbf{C})$, and activity (A). Further, data sources are denoted by the by sub indices denoting self-reports $(s r)$, fitbit $(f b)$, smartphone $(s p)$, and other passive sensing modalities $(p s)$.

Switzerland Dataset (CH-Dataset): We used a mobile sensing dataset called Bites' $n$ 'Bits collected in our group's previous work $[5,13]$. It contains smartphone sensor data, self-reported data, and activity data of fitbit wearables from 122 students of a Swiss university. The smartphone application allowed users to self-report details regarding eating events in-situ ( $\mathbf{T}$ : time of eating; $\mathbf{C}_{s r}$ : social context of eating, food type, concurrent activities, etc.). Further, their activity levels were captured using a fitbit wearable $\left(\mathbf{A}_{f b}\right.$ : step count, physical activity level), and activity level features were derived using the minutes spent on each of the four levels: sedentary, lightly active, fairly active, and very active. Moreover, passive sensing data regarding the context such as location were captured $\left(\mathbf{C}_{p s}\right)$. In the final dataset, there are 4448 reports (3414 meals, 1034 snacks). All the participants in the study were between $18-26$ in age, with a mean age of 20.5 years, and there were $65 \%$ men and $35 \%$ women.

Mexico Dataset (MX-Dataset): We collected a dataset from 84 university student in San Luis Potosi, Mexico using the same approach described in [5]. The dataset had self-reported features similar to $\mathrm{CH}$-Dataset $\left(\mathbf{C}_{s r}\right.$ and $\mathbf{T}$ feature groups), and instead of the FitBit wearable (which we left out for cost reasons), activity levels of students were captured using the phone accelerometer $\left(\mathbf{A}_{s p}: \mathrm{x}, \mathrm{y}\right.$, and $\mathrm{z}$ axis values of the accelerometer). Further, this dataset contained additional features about the participant context ( $\mathbf{C}_{p s}$ : app usage, radius of gyration, screen/battery charging events). The dataset contained 3278 reports (1911 meals, 1367 snacks). The average age of study participants was 23.4 years, and the cohort had $44 \%$ men and $56 \%$ women. A more detailed feature summary with naming conventions is available in [25].

Data Pre-Processing: During the feature extraction phase, standard datasets were created with one entry per eating event using a similar procedure to that given in [5]. For both datasets, if the eating event occured at time $\mathrm{T}$, we aggregated sensing data from $\mathrm{T}-\alpha$ to $\mathrm{T}+\alpha\left(\alpha_{C H}=2\right.$ hours as chosen in [5], $\alpha_{M X}=30$ minutes, we

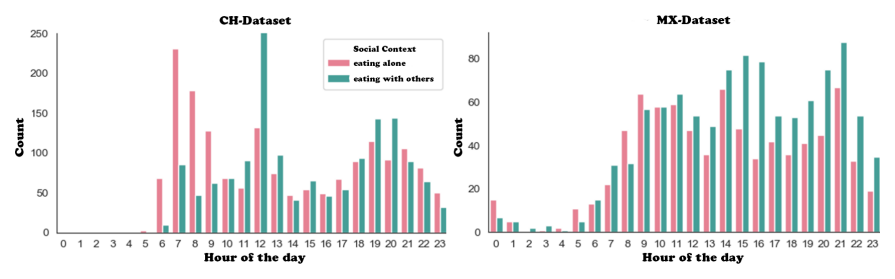

Figure 1: Temporal variation for social context of eating

present results for this value after examining performance of the model for different $\alpha$ ).

Activity (CH and MX Datasets): For both datasets, (CH-Dataset: step counts and activity levels from fitbit; MX-Dataset: phone accelerometer), initially, the physical activity related features were calculated for 10-minute slots throughout the day. For the $\mathrm{CH}$ Dataset: the total, median, mean, and standard deviation $(s d)$ values of these features were calculated for $\alpha_{C H}$ before (bef) and after (aft) each eating event using 10-minute based values. For MX-Dataset: features were derived from three axes of the accelerometer sensor using absolute (abs) values and real values for $\alpha_{M X}$ before (bef) and after ( $a f t$ ) each eating event. Then, for the time window corresponding to the eating event, mean of feature values was calculated using 10-minute based values.

Apps (MX-Dataset): We selected the ten most frequently used apps in the dataset. Then, during the eating time window, we determined whether each of the apps have been used or not, hence resulting in binary values for all app related features.

Location (CH and MX Datasets): using location traces, we calculated radius of gyration $[3,41]$ within the time period associated to the eating episode.

Screen (MX-Dataset): using screen-on/off time slots, we calculated the screen-on time during the hour of consideration, and also the number of times the screen was turned on, similar to prior literature [2].

Battery (MX-Dataset): we calculated the average battery level during the hour of consideration and also whether any charging events were detected during the time of eating episode.

\section{METHODOLOGY AND RESULTS}

\subsection{Descriptive Analysis}

Temporal Variations. Figure 1 shows the temporal variation of the reported eating episodes for different social contexts of eating in both datasets. For both figures, three peaks can be seen that correspond to breakfast, lunch, and dinner, even though times at which these peaks occur are different in the two datasets. This could be due to cultural differences between the students in the two countries. Note that students in MX often live with their family while attending college, while this is less common with $\mathrm{CH}$ students. In the $\mathrm{CH}$-Dataset, the time period from 6.00AM to 9.00AM (breakfast) results in significantly high number of eating-alone episodes compared to eating-with-others episodes. However, in the MX-Dataset, breakfast peak occurs later closer to 9.00AM to 11.00AM and the differences in terms of social context of eating are minimal, even though it still favors eating-alone. In $\mathrm{CH}$-Dataset, a significantly high number of eating-with-others episodes are present during the 

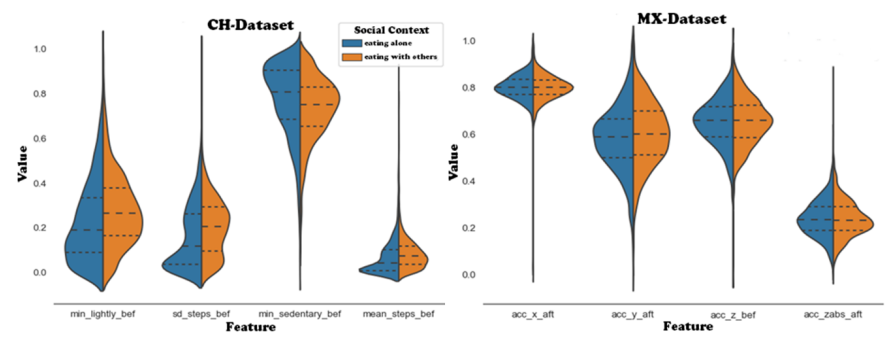

Figure 2: Violin plots for selected activity features

lunch peak, and a similar pattern can be seen in the MX-Dataset as well. This could be because students were eating in the university, with their friends. In the $\mathrm{CH}$-Dataset, dinner episodes are more or less even in terms of social context. However, the MX-Dataset show that even the dinner episodes are reported in highly social contexts, again partly explained for the living- with-parents situation. Hence, as a summary, Swiss students have reported high number of eatingalone episodes for breakfast, high number of eating-with-others episodes for lunch, and evenly distributed dinner episodes. For the MX students, except for the slight lean towards eating-alone in the morning, most other eating episodes have been reported to be with others. This could also be justified by prior research in psychology that has shown that Mexicans are highly social [28].

Passive Activity Variations. Figure 2 shows differences in distributions of sensed activity levels for the two social contexts of eating, for few selected features (due to space limitations) using Violin plots [1]. In the $\mathrm{CH}$-Dataset, activity level features captured using the wearable show significant mean differences for all four features shown here. For example, the distributions of feature mean steps bef (see Section 2 and [25] for feature descriptions) are different in terms of the shape, where eating-alone distribution is highly skewed towards lower values, meaning that activity levels are low for eating-alone episodes. A similar pattern can be seen for features such as min lightly bef and sd steps bef. The feature min sedentary bef, that corresponds to time spent in sedentary state, show higher values for eating-alone episodes. As a summary, lower physical activity levels around eating episodes correspond to eating-alone in the $\mathrm{CH}$-Dataset. This is consistent with prior work that has shown that low physical activity levels correspond to less social contexts $[34,38]$. However, in the MX-Dataset, the activity levels were sensed via the smartphone, and the mean differences are smaller for both social contexts of eating. Hence, for the MX case, it is less clear whether there are significant activity level differences depending on eating social context. However, it should be noted that the features generated regarding activity levels (based on availability) from the two datasets are different (fitbit for $\mathrm{CH}$, phone accelerometer for $\mathrm{MX})$, and this could have influenced the results.

\subsection{Statistical Analysis}

Table 1 shows statistics such as t-statistic [22], p-value [15], and cohen's-d (effect size) [23] for all the features in both datasets for two groups: eating-alone and eating-with-others. The objective is to identify features that discriminate between the two social contexts. In the table, the features are ordered by the descending order of cohen's-d value. We calculated cohen's-d [31] to help understand the statistical significance of the features because p-values are not sufficiently informative $[24,39]$. To interpret cohen's-d, we used a commonly used rule-of-thumb: small effect size $=0.2$, medium effect size $=0.5$ and large effect size $=0.8$. Moreover, we calculated $95 \%$ confidence interval for cohen's-d. A confidence interval that includes zero suggests statistical non-significance [24].

In the $\mathrm{CH}$-Dataset, the feature with highest cohen's-d (medium effect size) is time since last meal, which is derived from temporal aspects regarding food consumption. Moreover, physical activity features such as min lightly bef, sd steps bef, min sedentary bef, and mean steps bef that were derived from activity level by users before the eating event show cohen's-d values larger than 0.2 . Moreover, all the features had confidence intervals that did not include zero. Other two features in the top ten are location and time, that also have cohen's-d values larger than 0.2 . As a summary, the $\mathrm{CH}$ Dataset contained several features derived from fitbit that show discriminating signs between eating-alone and eating-with-others episodes.

When considering the MX-Dataset, the feature with highest cohen's-d (0.37) was location. However, the only other feature from this dataset that had a cohen's-d higher than small effect size was time (cohen's-d = 0.25). Even though several passive sensing features related to activity levels were in the top ten, only two features (acc y aft and acc $z$ abs aft) had confidence intervals that did not include zero. Another passive interaction sensing modality that had a closer to small effect size was charging events with a cohen's-d of 0.16 . Moreover, two app usage related passive sensing features (app google search and app spotify) were in the top ten. However, both these had cohen's-d confidence intervals including zero. As a summary, these results show that passive sensing features that quantify the activity levels, time of eating, and location have shown signs of discriminating capability between eating-alone and eatingwith-others episodes in both datasets.

\subsection{Inferring Eating Episodes: Alone or With Others}

The goal of the 2-class inference task was to use different subsets of features in the training set, and calculate the accuracy, precision, and recall. The target binary variable was eating-alone vs. eatingwith-others, which indicates this fundamental aspect of eating. Moreover, we used random forest classifiers (RF) with ntree values between 100 - 150. We followed leave k-participants out strategy for all the experiments when preparing the dataset, where training and testing splits did not have data from the same user. Moreover, when preparing the dataset, we made sure that the classes are balanced by upsampling the minority classes to get balanced datasets (similar to [5]). Further, we conducted the experiment for different feature groups, and feature group combinations such as (a) A: these are features generated using the activity data from fitbit wearables and smartphones. Features in this group are passively sensed, hence not needing any user interaction; (b) A+T: when temporal features such as time of the day and time since last food intake are combined with activity data, it provides a temporal variation of the activity levels; and (c) $\mathrm{A}+\mathrm{T}+\mathrm{C}_{p s}$ : this feature group contains only passive sensing features that are activity data and contextual data, and time 
Table 1: Comparative statistics of top 10 features across classes eating-alone and eating-with-others: $t$-statistic, $p$-value, and cohen's-d with 95\% confidence intervals. Features are sorted based on the decreasing order of cohen's-d; 95\% confidence interval of cohen's-d CI includes $0={ }^{x}$; when considering $\mathbf{p}$-values, $\mathbf{p}<0.0001={ }^{*}, \mathbf{p}<0.001={ }^{* *}, \mathbf{p}<0.01={ }^{* * *}$.

\begin{tabular}{|c|c|c|c|c|c|c|c|}
\hline \multirow[t]{2}{*}{ Feature } & \multirow[t]{2}{*}{ Feature Group } & \multicolumn{2}{|c|}{ CH-Dataset } & \multirow[t]{2}{*}{ Feature } & \multirow[t]{2}{*}{ Feature Group } & \multicolumn{2}{|c|}{ MX-Dataset } \\
\hline & & cohen's-d & t-statistic & & & cohen's-d & t-statistic \\
\hline time since last meal & $\mathrm{T}$ & 0.57276 & $16.77194^{*}$ & location & $\mathrm{C}_{p s}$ & 0.37116 & $5.86325^{*}$ \\
\hline min lightly bef & $\mathrm{A}_{f b}$ & 0.32612 & $9.54787^{*}$ & time & $\mathrm{T}$ & 0.25114 & $4.02973^{*}$ \\
\hline sd steps bef & $\mathrm{A}_{f b}$ & 0.31644 & $9.27218^{*}$ & charging event & $\mathrm{C}_{p s}$ & 0.16151 & $2.70769^{\star *}$ \\
\hline location & $\mathrm{C}_{p s}$ & 0.26917 & $7.87712^{*}$ & acc y aft & $\mathrm{A}_{s p}$ & 0.15354 & 2.45258 \\
\hline min sed bef & $\mathrm{A}_{f b}$ & 0.26841 & $7.86013^{*}$ & acc y abs aft & $\mathrm{A}_{s p}$ & 0.12842 & 2.05463 \\
\hline time & $\mathrm{T}$ & 0.21020 & $6.15727^{*}$ & app google search & $\mathrm{C}_{p s}$ & $0.11903^{x}$ & 1.87712 \\
\hline concurrent activity & $\mathrm{C}_{s r}$ & 0.20756 & $6.09132^{*}$ & acc $z$ bef & $\mathrm{A}_{s p}$ & $0.11629^{x}$ & 1.86609 \\
\hline mean steps bef & $\mathrm{A}_{f b}$ & 0.20101 & $5.88661^{*}$ & acc $\mathrm{z}$ abs aft & $\mathrm{A}_{s p}$ & $0.10989^{x}$ & 1.76660 \\
\hline tot steps bef & $\mathrm{A}_{f b}$ & 0.20079 & $5.88032^{*}$ & screen on & $\mathrm{C}_{p s}$ & $0.09536^{x}$ & 1.51781 \\
\hline min lightly aft & $\mathrm{A}_{f b}$ & 0.12267 & $3.59117^{* * *}$ & app spotify & $\mathrm{C}_{p s}$ & $0.09453^{x}$ & 1.48093 \\
\hline
\end{tabular}

of eating; (d) $\mathrm{A}+\mathrm{T}+\mathrm{C}_{s r}$ : this feature group contains only passive activity sensing features and contextual data that were self reported; (e) $\mathrm{A}+\mathrm{T}+\mathrm{C}_{p s}+\mathrm{C}_{s r}$ : this combines all the available passive sensing and self-report features from wearables and smartphones to conduct the inference task. The baseline for experiments is $50 \%$ since the classes were balanced in the inference task.

Results are summarized in Table 2. As shown there, by only using activity data captured via wearables and smartphones, the models reached accuracies of $75.54 \%$ and $70.57 \%$ for the $\mathrm{CH}$-Dataset and MX-Dataset, respectively. These accuracies are considerably increased when using temporal features. The $\mathrm{A}+\mathrm{T}+\mathrm{C}_{p s}$ feature group shows how models that primarily use passive sensing data (even though $\mathrm{T}$ is self-reported, prior work has shown that the time of eating can be inferred to some extent with mobile sensing $[4,36]$. Hence, this feature group combination could be considered as nearpassive.) without high user interaction to input details regarding details regarding food type or calorie levels, can infer eating social context with accuracies of $80.89 \%$ and $77.73 \%$ for $\mathrm{CH}$-Dataset and MX-Dataset, respectively. For this feature group, features such as time, time since last meal, location, and other activity related features were among the top five for both datasets, when considering feature importance values derived from the RFs. Moreover, when all the features used in mobile food diaries with sensing capabilities are considered, the inference accuracies reached higher values of $90.88 \%$ and $84.08 \%$ for the $\mathrm{CH}$ and MX datasets, respectively. These results show that precise wearable sensing and even smartphone sensing (that can be obtained regardless of the smartphone type or brand) are both useful to infer eating-alone vs. eating-withothers episodes. This could be seen as a first step towards enabling holistic mobile food diaries with reduced user burden, by inferring attributes that are typically captured with self-reports.

\section{CONCLUSION}

In this paper, we investigated whether mobile sensing features could help identify the social context of eating episodes, using datasets from two countries (Switzerland and Mexico). We identified dataset features that help discriminate between two sociability levels (eating-alone and eating-with-others), and finally, we tackled a novel ubicomp task to infer eating-alone vs. eating-with-others with accuracies of the range $77 \%-81 \%$ by using passive sensing
Table 2: Eating-alone vs. eating-with-others inference task

\begin{tabular}{|c|c|c|c|c|}
\hline & Feature Group & Accuracy & Precision & Recall \\
\hline & Baseline & $50.00 \%$ & - & - \\
\hline \multirow{5}{*}{ 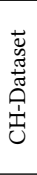 } & $A_{f b}$ & $75.54 \%$ & $75.52 \%$ & $75.53 \%$ \\
\hline & $\mathrm{A}_{f b}+\mathrm{T}$ & $80.31 \%$ & $80.35 \%$ & $80.29 \%$ \\
\hline & $\mathrm{A}_{f b}+\mathrm{T}+\mathrm{C}_{p s}$ & $80.89 \%$ & $80.96 \%$ & $80.90 \%$ \\
\hline & $\mathrm{A}_{f b}+\mathrm{T}+\mathrm{C}_{s r}$ & $89.97 \%$ & $90.31 \%$ & $89.56 \%$ \\
\hline & $\mathrm{A}_{f b}+\mathrm{T}+\mathrm{C}_{p s}+\mathrm{C}_{s r}$ & $90.88 \%$ & $91.11 \%$ & $90.69 \%$ \\
\hline \multirow{5}{*}{ 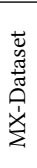 } & $\mathrm{A}_{s p}$ & $70.57 \%$ & $71.11 \%$ & $71.10 \%$ \\
\hline & $\mathrm{A}_{s p}+\mathrm{T}$ & $72.30 \%$ & $73.03 \%$ & $72.92 \%$ \\
\hline & $\mathrm{A}_{s p}+\mathrm{T}+\mathrm{C}_{p s}$ & $77.73 \%$ & $77.74 \%$ & $77.77 \%$ \\
\hline & $\mathrm{A}_{s p}+\mathrm{T}+\mathrm{C}_{s r}$ & $78.29 \%$ & $78.59 \%$ & $78.31 \%$ \\
\hline & $\mathrm{A}_{s p}+\mathrm{T}+\mathrm{C}_{p s}+\mathrm{C}_{s r}$ & $84.08 \%$ & $84.24 \%$ & $84.03 \%$ \\
\hline
\end{tabular}

data from wearables and smartphones. Even when only activity related data sensed via wearables and smartphones are considered, binary sociability can be inferred with accuracies of $70.57 \%$ and $75.54 \%$ for the MX-Dataset and CH-Dataset, respectively. These accuracies can be increased to the range of $84 \%-91 \%$ by combining other self-reported attributes. These results show initial progress towards inferring traditionally self-reported attributes such as social context of eating, which is useful to design mobile food diaries that reduce user burden and provide context-aware functionalities. This analysis could also be a first step towards understanding more complex social contexts (with friends or with family), which remain open for future investigation. Finally, it would also be interesting to examine how approaches such as transfer learning can be used to examine attributes from datasets of similar nature (e.g. about food consumption behavior), but have certain differences (e.g. passive sensing data captured from Switzerland and Mexico using slightly different approaches).

\section{ACKNOWLEDGMENTS}

This work was funded by the European Union's Horizon 2020 WeNet project, under grant agreement 823783. D. Gatica-Perez acknowledges the former support of EPFL Integrative Food and Nutrition Center, through the Understanding Eating Routines in Context project. 


\section{REFERENCES}

[1] 2020. seaborn violinplot. Retrieved May 28, 2020 from https://seaborn.pydata. org/generated/seaborn.violinplot.html

[2] Saeed Abdullah, Elizabeth L. Murnane, Mark Matthews, Matthew Kay, Julie A. Kientz, Geri Gay, and Tanzeem Choudhury. 2016. Cognitive Rhythms: Unobtrusive and Continuous Sensing of Alertness Using a Mobile Phone. In Proceedings of the 2016 ACM International Foint Conference on Pervasive and Ubiquitous Computing (Heidelberg, Germany) (UbiComp '16). ACM, New York, NY, USA, 178-189.

[3] Gianni Barlacchi, Christos Perentis, Abhinav Mehrotra, Mirco Musolesi, and Bruno Lepri. 2017. Are you getting sick? Predicting influenza-like symptoms using human mobility behaviors. EPf Data Science 6 (12 2017), 27.

[4] Abdelkareem Bedri, Richard Li, Malcolm Haynes, Raj Prateek Kosaraju, Ishaan Grover, Temiloluwa Prioleau, Min Yan Beh, Mayank Goel, Thad Starner, and Gregory Abowd. 2017. EarBit: Using Wearable Sensors to Detect Eating Episodes in Unconstrained Environments. Proc. ACM Interact. Mob. Wearable Ubiquitous Technol. 1, 3, Article 37 (Sept. 2017), 20 pages.

[5] Joan-Isaac Biel, Nathalie Martin, David Labbe, and Daniel Gatica-Perez. 2018. Bites'n'Bits: Inferring Eating Behavior from Contextual Mobile Data. Proc. ACM Interact. Mob. Wearable Ubiquitous Technol. 1, 4, Article 125 (Jan. 2018), 33 pages.

[6] Carole A. Bisogni, Laura Winter Falk, Elizabeth Madore, Christine E. Blake, Margaret Jastran, Jeffery Sobal, and Carol M. Devine. 2007. Dimensions of everyday eating and drinking episodes. Appetite 48, 2 (2007), 218 - 231.

[7] Peggy Bongers, Anita Jansen, Remco Havermans, Anne Roefs, and Chantal Nederkoorn. 2013. Happy eating. The underestimated role of overeating in a positive mood. Appetite 67 (2013), $74-80$

[8] Hilde Bruch. 1964. Psychological Aspects of Overeating And Obesity. Psychosomatics 5, 5 (1964), 269 - 274

[9] E. A. Carroll, M. Czerwinski, A. Roseway, A. Kapoor, P. Johns, K. Rowan, and M. C. Schraefel. 2013. Food and Mood: Just-in-Time Support for Emotional Eating. In 2013 Humaine Association Conference on Affective Computing and Intelligent Interaction. $252-257$.

[10] J Chua, Stephen Touyz, and AJ Hill. 2004. Negative mood-induced overeating in obese binge eaters: An experimental study. International journal of obesity and related metabolic disorders : journal of the International Association for the Study of Obesity 28 (05 2004), 606-10.

[11] Tegan Cruwys, Kirsten E. Bevelander, and Roel C.J. Hermans. 2015. Social modeling of eating: A review of when and why social influence affects food intake and choice. Appetite 86 (2015), 3 - 18. Social Influences on Eating.

[12] Robin Ian MacDonald Dunbar. 2017. Breaking Bread: the Functions of Social Eating. Adaptive Human Behavior and Physiology 3 (2017), $198-211$.

[13] Daniel Gatica-Perez, Joan-Isaac Biel, David Labbe, and Nathalie Martin. 2019 Discovering eating routines in context with a smartphone app. In UbiComp/ISWC Adjunct.

[14] Luke Gemming, Aiden Doherty, Jennifer Utter, Emma Shields, and Cliona Ni Mhurchu. 2015. The use of a wearable camera to capture and categorise the environmental and social context of self-identified eating episodes. Appetite 92 (2015), $118-125$.

[15] Greenland Sander, Senn Stephen J., Rothman Kenneth J., Carlin John B., Poole Charles, Goodman Steven N., and Altman Douglas G. 2016. Statistical tests, P values, confidence intervals, and power: a guide to misinterpretations. European fournal of Epidemiology 31, 4 (2016), 337-350.

[16] Juliet Haarman, Roelof de Vries, Emiel Harmsen, Hermie Hermens, and Dirk Heylen. 2020. Sensory Interactive Table (SIT) - Development of a Measurement Instrument to Support Healthy Eating in a Social Dining Setting. Sensors 20 (05 2020), 2636

[17] C.P. Herman, J. Polivy, and T. Leone. 2005. 6 - The psychology of overeating. In Food, Diet and Obesity, David J. Mela (Ed.). Woodhead Publishing, 115 - 136.

[18] C. P. Herman and J. Polivy. 2003. Dieting as an exercise in behavioral economics Time and decision: Economic and psychological perspectives on intertemporal choice (2003).

[19] Marion M. Hetherington, Annie S. Anderson, Geraldine N.M. Norton, and Lisa Newson. 2006. Situational effects on meal intake: A comparison of eating alone and eating with others. Physiology/\& Behavior 88, 4 (2006), 498-505.

[20] Suzanne Higgs and Jason Thomas. 2016. Social influences on eating. Current Opinion in Behavioral Sciences 9 (2016), 1 - 6. https://doi.org/10.1016/j.cobeha 2015.10.005 Diet, behavior and brain function.

[21] Thivya Kandappu, Abhinav Mehrotra, Archan Misra, Mirco Musolesi, Shih-Fen Cheng, and Lakmal Meegahapola. 2020. PokeME: Applying Context-Driven
Notifications to Increase Worker Engagement in Mobile Crowd-Sourcing. In Proceedings of the 2020 Conference on Human Information Interaction and Retrieval (Vancouver BC, Canada) (CHIIR '20). 3-12.

[22] Tae Kim. 2015. T test as a parametric statistic. Korean fournal of Anesthesiology 68 (11 2015), 540

[23] Daniël Lakens. 2013. Lakens D. Calculating and reporting effect sizes to facilitate cumulative science: a practical primer for t-tests and ANOVAs. Front Psychol 4: 863. Frontiers in psychology 4 (11 2013), 863.

[24] Dong Kyu Lee. 2016. Alternatives to P value: confidence interval and effect size. In Korean journal of anesthesiology.

[25] Lakmal Meegahapola, Salvador Ruiz-Correa, and Daniel Gatica-Perez. 2020. Protecting Mobile Food Diaries from Getting too Personal. In Proceedings of the 19th International Conference on Mobile and Ubiquitous Multimedia (Essen, Germany) (MUM '20). Association for Computing Machinery, New York, NY, USA.

[26] Weiqing Min, Shuqiang Jiang, Linhu Liu, Yong Rui, and Ramesh Jain. 2019. A Survey on Food Computing. ACM Comput. Surv. 52, 5, Article 92 (Sept. 2019), 36 pages.

[27] Mobius. 2019. 11 surprising mobile health statistics. Retrieved April 28, 2020 from https://www.mobius.md/blog/2019/03/11-mobile-health-statistics/

[28] Ramírez-Esparza Nairán, Matthias R. Mehl, Javier Álvarez Bermúdez, and James W. Pennebaker. 2009. Are Mexicans more or less sociable than Americans? Insights from a naturalistic observation study. fournal of Research in Personality 43, 1 (2009), 1 - 7 .

[29] K.A. Patel and D.G. Schlundt. 2001. Impact of moods and social context on eating behavior. Appetite 36, 2 (2001), 111 - 118

[30] Hannah Payne, Cameron Lister, Joshua West, and Jay Bernhardt. 2015. Behavioral Functionality of Mobile Apps in Health Interventions: A Systematic Review of the Literature. FMIR mHealth and uHealth 3 (02 2015), e20.

[31] Marnie E. Rice and Grant T. Harris. 2005. Comparing Effect Sizes in Follow-Up Studies: ROC Area, Cohen's d, and r. Law and Human Behavior 29, 5 (01 Oct 2005), 615-620

[32] D. Santani, T. Do, F. Labhart, S. Landolt, E. Kuntsche, and D. Gatica-Perez. 2018. DrinkSense: Characterizing Youth Drinking Behavior Using Smartphones. IEEE Transactions on Mobile Computing 17, 10 (2018), 2279-2292.

[33] Edmund Seto, Jenna Hua, Lemuel Wu, Victor Shia, Sue Eom, May Wang, and Yan Li. 2016. Models of Individual Dietary Behavior Based on Smartphone Data: The Influence of Routine, Physical Activity, Emotion, and Food Environment. PLOS ONE 11 (04 2016).

[34] Rachel Shelton, Lorna Mcneill, Elaine Puleo, Kathleen Wolin, Karen Emmons, and Gary Bennett. 2011. The Association Between Social Factors and Physical Activity Among Low-Income Adults Living in Public Housing. American journal of public health 101 (02 2011), 2102-10.

[35] Christine Sheppard-Sawyer, Richard McNally, and Jennifer Fischer. 2000. Filminduced sadness as a trigger for disinhibited eating. The International journal of eating disorders 28 (10 2000), 215-20.

[36] Edison Thomaz, Irfan Essa, and Gregory D. Abowd. 2015. A Practical Approach for Recognizing Eating Moments with Wrist-Mounted Inertial Sensing. In Proceedings of the 2015 ACM International foint Conference on Pervasive and Ubiquitous Computing (Osaka, Japan) (UbiComp '15). 1029-1040.

[37] Bengisu Tulu, Carolina Ruiz, Joshua Allard, Joseph Acheson, Andrew Busch, Andrew Roskusku, Gage Heeringa, Victor Jaskula, Jessica Oleski, and Sherry Pagoto. 2017. SlipBuddy: A Mobile Health Intervention to Prevent Overeating. (01 2017).

[38] Staphanie Ward, Mathieu Baelanger, Denise Donovan, and Natalie Carrier. 2016. Relationship between eating behaviors and physical activity of preschoolers and their peers: A systematic review. International fournal of Behavioral Nutrition and Physical Activity 13 (12 2016).

[39] Koji Yatani. 2016. Effect Sizes and Power Analysis in HCI. Springer International Publishing, Cham, 87-110.

[40] Luke Yates and Alan Warde. 2017. Eating together and eating alone: meal arrangements in British households. The British fournal of Sociology 68, 1 (2017), 97-118.

[41] Yang Yue, Tian Lan, Anthony G.O. Yeh, and Qing-Quan Li. 2014. Zooming into individuals to understand the collective: A review of trajectory-based travel behaviour studies. Travel Behaviour and Society 1, 2 (2014), $69-78$.

[42] Lydia Zepeda and David Deal. 2008. Think before you eat: photographic food diaries as intervention tools to change dietary decision making and attitudes. International fournal of Consumer Studies 32, 6 (2008), 692-698. 